\title{
Silica Nano Powder Formation using ICP Plasma and Evaluation of its Adsorption Ability for Biotic Substances
}

\author{
Aya Satomi, Mitsuyoshi Mio ${ }^{\ddagger}$, Kunihito Tanaka and Masuhiro Kogoma \\ Department of Chemistry, Faculty of Science and Technology, Sophia University, \\ 7-1 Kioicho, Chiyoda-ku, Tokyo, Japan \\ ${ }^{\ddagger}$ School of Pharmacy, Shujitsu University, 1-6-1 Nishikawara, Okayama, Japan
}

\begin{abstract}
We synthesized silica nano powder using ICP plasma and evaluated its adsorption ability for biotic substances such as proteins and molecular histamine. The silica particles with negative electric charge adsorbed cytochrome $\mathrm{C}$ and histamine which have a positive electric charge. On the other hand, OVA which has a negative electric charge was not adsorbed. We considered that electrostatic interactions dominate the adsorption process between silica particles and biotic substances.
\end{abstract}

Keywords: silica nano powder, ICP plasma, adsorption, histamine

\section{Introduction}

Silica powder is generally known as a safe substance for skin, so it is used as a material for a cosmetic foundation. Also it is well known that silica particles have high adsorption ability, so they are used as adsorbents. [1]

Then, we planned to synthesize silica nano powder using ICP plasma. ICP plasma can make an ultrahigh temperature condition, so it is used for the pyrolysis synthesis of inorganic oxides. [2] The particles formed under the ultrahigh temperature condition are rapidly quenched. As a result, the particles which have a small mean diameter and a large specific surface area can be formed using ICP plasma. It is thought that silica particles which have a large specific surface area have an advantage of better adsorption ability for various substances.

In this study, we synthesized silica nano powder using ICP plasma and evaluated its particle adsorption ability for some biotic substances. The aims of this study are to collect information of adsorption ability of silica particles for various biotic substances and to determine the mechanism of adsorption between silica particles and biotic substances clearly. Then, we would like to consider an application of such silica particles to be adsorbents for proteins or biotic substances which are harmful to humans.

\section{Experimental}

2.1. Plasma reactor and the powder formation

Figure 1 shows the ICP plasma reactor used in this study. It can produce temperatures higher than $5000 \mathrm{~K}$. The monomer vapor (chlorotrimethylsilane, CTMS) was mixed with carrier gas $\left(\mathrm{Ar}-\mathrm{O}_{2}\right.$ mixture) and introduced to the plasma flame

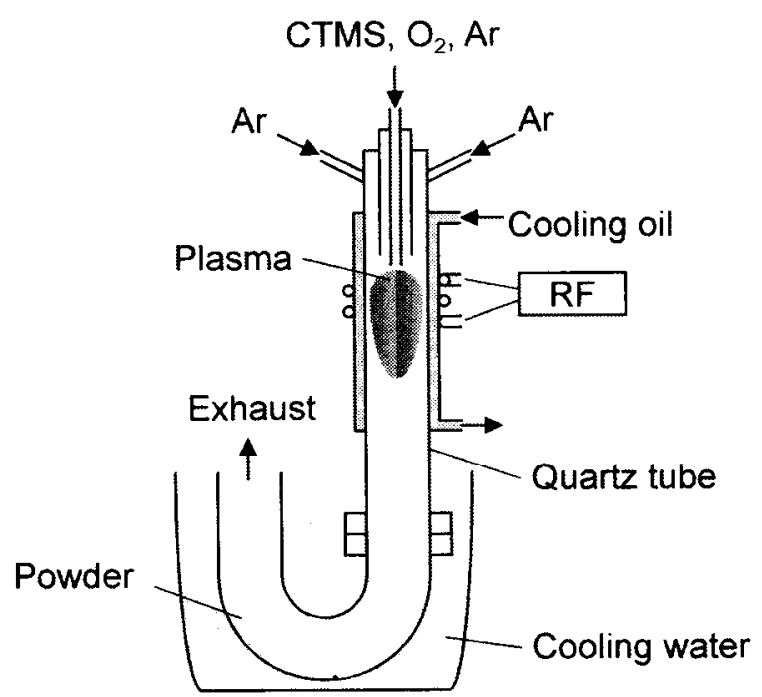

Fig. 1. Schematic diagram of the ICP plasma reactor. 


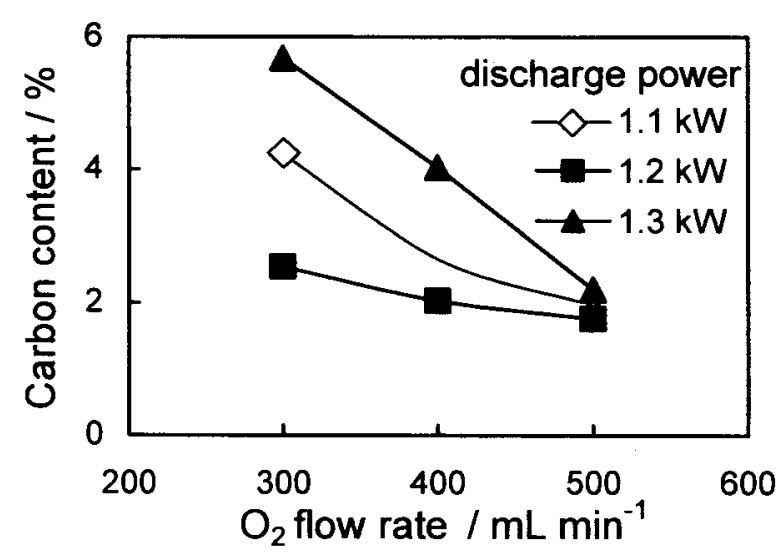

Fig. 2. Carbon content in silica powder measured by XPS.

through the center inlet tube. Then, the monomer vapor was thermally decomposed, and silica particles were formed. Produced particles were collected on the wall of the recovery part.

The power source used in this study generates $27.12 \mathrm{MHz}$. The discharge power is $1.1-1.3 \mathrm{~kW}$. The total Ar and $\mathrm{O}_{2}$ flow rates are $24.3 \mathrm{~L} \mathrm{~min}^{-1}$ and $300-500 \mathrm{~mL} \mathrm{~min}^{-1}$; the CTMS flow rate is $18 \mathrm{~mL}$ $\mathrm{min}^{-1}$. The quartz tube reactor is cooled by circulation of fluorinated organic oil.

\subsection{Evaluation of properties of silica powders}

Elemental analysis of formed silica powders was done by XPS (Phi 5800ci) analysis. The diameter measurement of the particles was done by SEM (Hitachi, S-430). The functional groups and specific surface areas of particles were measured by FT-IR (JASCO, FT/IR-4100) and BET.

\subsection{Evaluation of adsorption ability of silica parti-} cles for biotic substances

In evaluations of the adsorption ability of silica particles for biotic substances, $10 \mathrm{mg}$ of silica powder was suspended in $0.9 \mathrm{~mL}$ of $10 \mathrm{mM}$ MES (2-morpholinoethanesulfonic acid) buffer ( $\mathrm{pH} 6.0$, Nacalai Tesque) and dispersed well under ultrasonic irradiation for $30 \mathrm{~min}$ using a bath-type sonicator (Tokyo Choonpa Giken, US-C-600). Then, $0.1 \mathrm{~mL}$ of biotic substance solution was added; incubation was carried out at $37{ }^{\circ} \mathrm{C}$ for $30 \mathrm{~min}$. Afterward, silica particles were precipitated by a centrifugation (Eppendorf, Centrifuge 5418) at $16800 \times \mathrm{g}$ for $30 \mathrm{~min}$. UV absorption spectra of the supernatant were measured by means of a UV-spectrophotometer (JASCO, V-650).

Until now, ovalbumin (OVA; Sigma), bovine serum albumin (BSA; Sigma) and cytochrome C

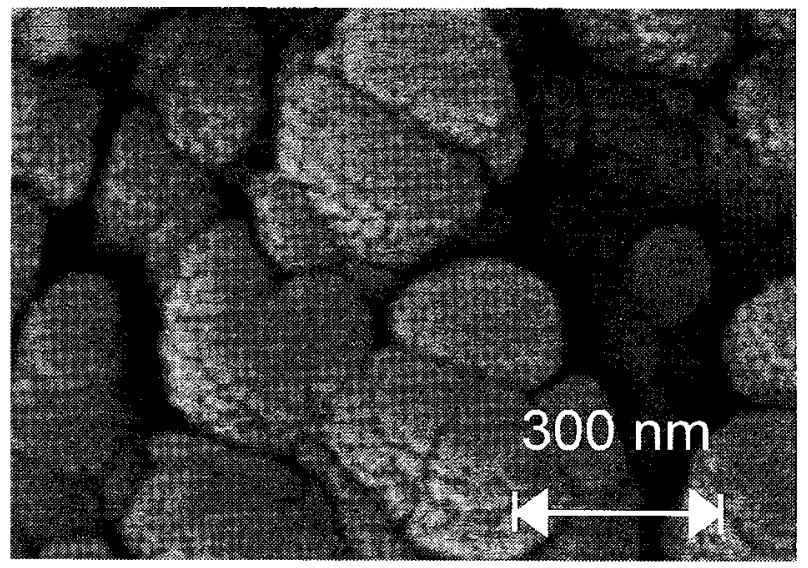

Fig. 3. SEM image of synthesized silica powder.

(Sigma) have been used as protein models. Whether protein was adsorbed by silica particles or not was evaluated by comparing the absorption at $280 \mathrm{~nm}$ of protein solution in the presence and absence of silica particles.

Moreover, histamine (Sigma) has been used as a model of a neurotransmitter. Whether histamine was adsorbed by silica particles or not was evaluated by colorimetric determination method. By comparing the absorption at $460 \mathrm{~nm}$ of histamine solution using histamine quantitative kit (Kikkoman, checkcolor histamine), we evaluated the adsorption ability of silica for histamine.

For evaluation of surface electric charges of particles of silica and various proteins and histamine, we used a zeta potential analyzer (Microtec Nicion, ZEECOM ZX2000). Each measurement was carried out under pH 6 using $10 \mathrm{mM}$ MES.

\section{Results and discussion}

\subsection{Powder formation using ICP plasma}

Figure 2 shows the carbon content in the formed silica powder measured by XPS. The carbon content in silica was decreased with increasing of $\mathrm{O}_{2}$ flow rate. This is because CTMS was better oxidized by sufficient oxygen, so impurities like carbon were reduced. We could synthesize silica powder with $2 \%$ of carbon contamination at 500 $\mathrm{mL} \min ^{-1}$ of $\mathrm{O}_{2}$ flow rate. Chlorine was undetected in all conditions.

Figure 3 shows a SEM image of silica powder. Diameters of the powder particles measured by SEM were about $100-300 \mathrm{~nm}$. But most large particles were $2^{\text {nd }}$ order particles which were composed of small $1^{\text {st }}$ order particles. The diameters of $1^{\text {st }}$ order particles were $30-50 \mathrm{~nm}$.

Figure 4 shows the mean diameter of $2^{\text {nd }}$ order 


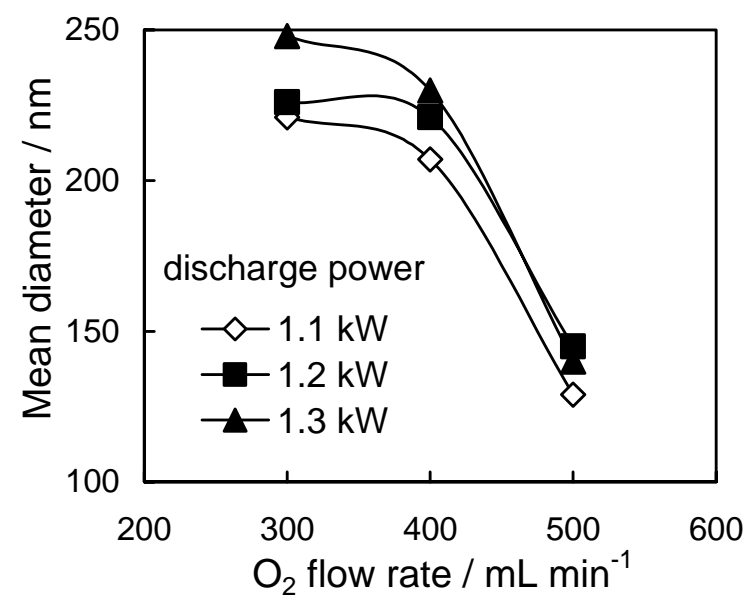

Fig. 4. Mean diameter of silica particles measured by SEM.

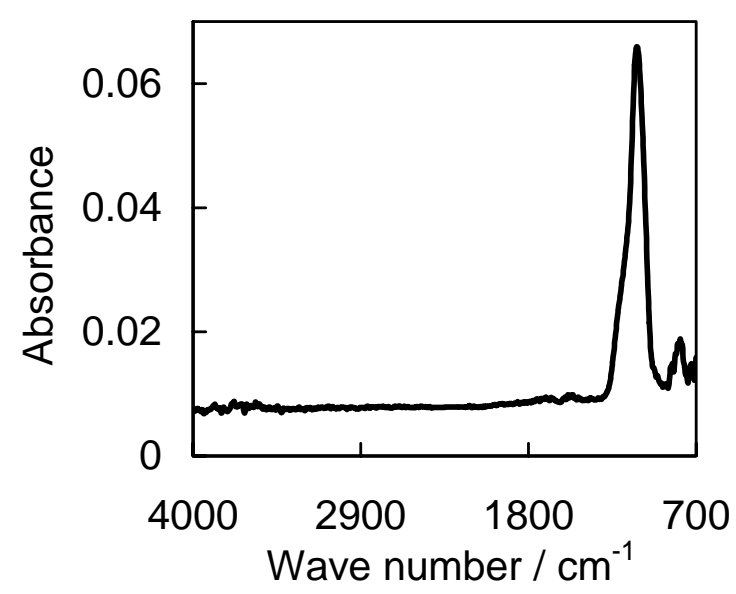

Fig. 5. IR absorption spectra of silica powders.

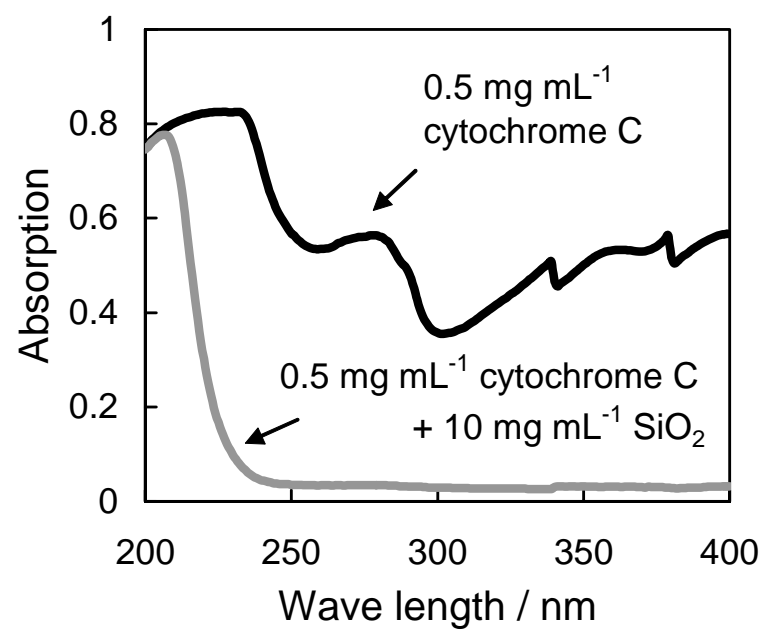

Fig. 6. UV spectra of $0.5 \mathrm{mg} \mathrm{mL}^{-1}$ cytochrome $\mathrm{C}$ with $10 \mathrm{mg} \mathrm{mL}^{-1}$ of silica particles. particles of silica measured by SEM. The mean diameter of silica particles was decreased with increasing of $\mathrm{O}_{2}$ flow rate. This is because rich oxygen in the plasma flame causes rapid cooling effect for the synthesized particles, so formed particles did not easily agglutinate with each other.

Figure 5 shows the IR spectrum of silica powder. Formed silica powder has Si-O-Si peak at $1050 \mathrm{~cm}^{-1}$, but no peak at about $3500 \mathrm{~cm}^{-1}$, where $\mathrm{OH}$ peak is. This spectrum means that the formed silica powder is almost pure.

The mean specific surface area of particles was about $170 \mathrm{~m}^{2} \mathrm{~g}^{-1}$ measured by BET method using $\mathrm{N}_{2}$. Both this result and the information of mean diameters measured by SEM image suggest that silica particles were not porous.

\subsection{Protein adsorption in buffer solution}

We evaluated formed silica particles' adsorption ability for various proteins. In the case of OVA, the UV absorption spectrum of it was not affected by $10 \mathrm{mg} \mathrm{mL}^{-1}$ of silica particles at $0.05-10 \mathrm{mg} / \mathrm{mL}$. This result means that the OVA was not adsorbed by silica particles at $\mathrm{pH} 6$.

On the other hand, in the cases of BSA and cytochrome $\mathrm{C}$, the UV absorption spectra were affected by silica particles. About BSA, the UV absorption of $0.05 \mathrm{mg} \mathrm{mL}^{-1}$ BSA at $280 \mathrm{~nm}$ was almost completely removed by addition of $10 \mathrm{mg}$ $\mathrm{mL}^{-1}$ of silica particles. Also in the cases of 0.25 $\mathrm{mg} \mathrm{mL}^{-1}$ and $0.5 \mathrm{mg} \mathrm{mL}^{-1}$ BSA, UV absorption at $280 \mathrm{~nm}$ was depressed by the addition of silica particles.

In the case of cytochrome $\mathrm{C}$, absorption of $0.5 \mathrm{mg}$ $\mathrm{mL}^{-1}$ Cytochrome $\mathrm{C}$ at ultra violet and visual

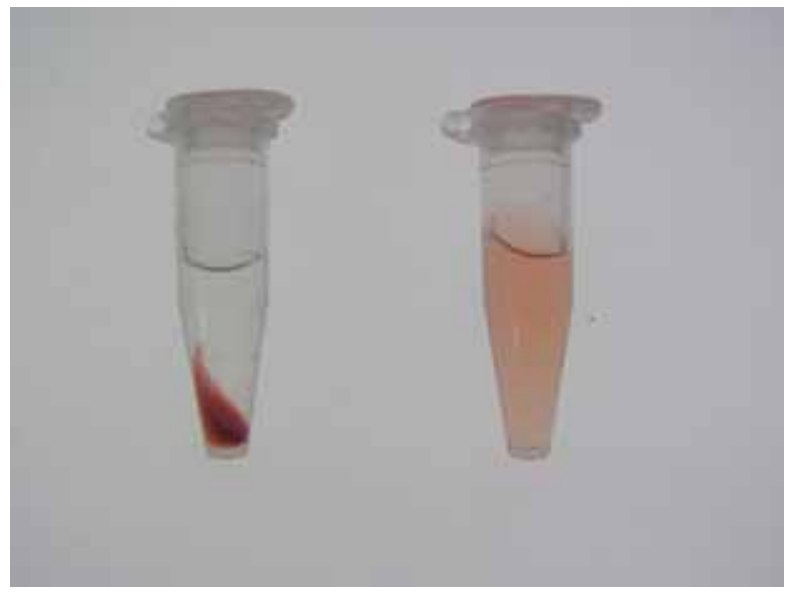

Fig. 7. The image of cytochrome $\mathrm{C}$ solution after incubation and precipitation. Left tube: $0.5 \mathrm{mg} \mathrm{mL}^{-1}$ cytochrome $\mathrm{C}$ with $10 \mathrm{mg} \mathrm{mL}^{-1}$ of silica particles, right tube: $0.5 \mathrm{mg} \mathrm{mL}^{-1}$ cytochrome C. 
light range was almost completely removed by addition of $10 \mathrm{mg} \mathrm{mL}^{-1}$ of silica particles (Figure 6).

The solution of cytochrome $\mathrm{C}$ has a red color, but the solution after incubation and precipitation with silica particles was clear and colorless (Figure 7). The change shows that $0.5 \mathrm{mg} \mathrm{mL}^{-1}$ cytochrome $\mathrm{C}$ was surely adsorbed by $10 \mathrm{mg}$ of silica particles.

\subsection{Histamine adsorption in buffer solution}

Likewise, we evaluated the adsorption ability of silica particles for histamine. Figure 8 shows the result of histamine adsorption experiments. At a histamine concentration of $0.05-6 \mathrm{mg} \mathrm{mL}^{-1}$, the amount of histamine adsorbed by silica particles was directly increased with increasing histamine concentration. The amount of adsorbed histamine was about $13 \%$ of the original amount of histamine in the buffer solution.

\subsection{Zeta potential}

To determine the mechanism of silica particle's adsorption ability for biotic substances, we measured the zeta potentials of silica particles and some biotic substances. Until now, zeta potentials of the following substances have been measured: OVA, histamine and silica particles synthesized by ICP plasma.

Figure 9 shows the distribution of zeta potentials of OVA and histamine. In the case of OVA, which was not adsorbed by silica particles, it had a negative surface electric charge in $\mathrm{pH} 6$. The distribution of zeta potential of OVA had a maximum value at about $-20 \mathrm{mV}$. On the other hand, in the case of histamine which was adsorbed by silica particles, it had a large distribution of zeta potential. The distribution of zeta potential of histamine had two peaks near $-5 \mathrm{mV}$ and $2 \mathrm{mV}$. Most particles of histamine had a negative surface electric charge, but about $12 \%$ of the particles had a positive surface electric charge.

Figure 10 shows the distribution of zeta potential of silica particles synthesized by ICP plasma. The distribution of zeta potential of silica particles had a maximum value at $-25 \mathrm{mV}$, and all particles had a negative surface electric charge.

\subsection{Discussion}

After evaluation of the adsorption ability of silica particles for various proteins, we see that cytochrome $\mathrm{C}$ and BSA were adsorbed by silica, but OVA was not adsorbed by silica.

The isoelectric points of cytochrome $\mathrm{C}$ and

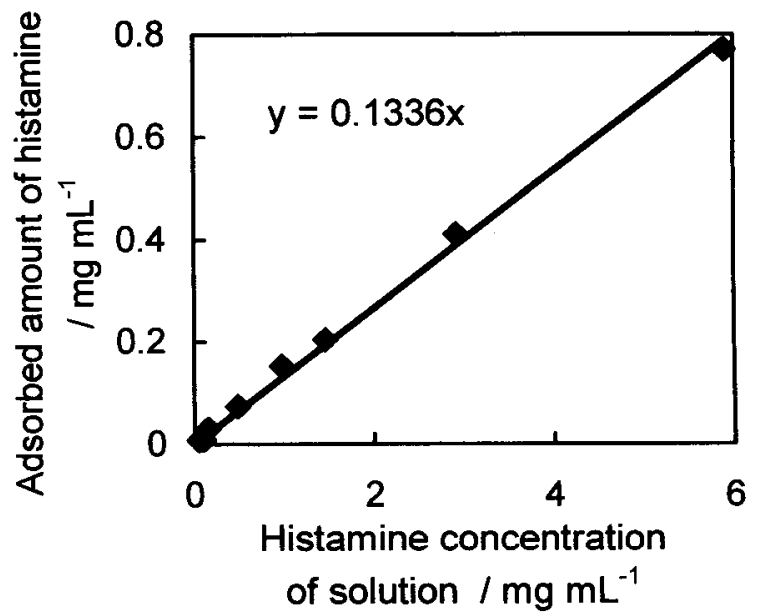

Fig. 8. The relation between histamine concentration of solution and amount of histamine adsorbed.

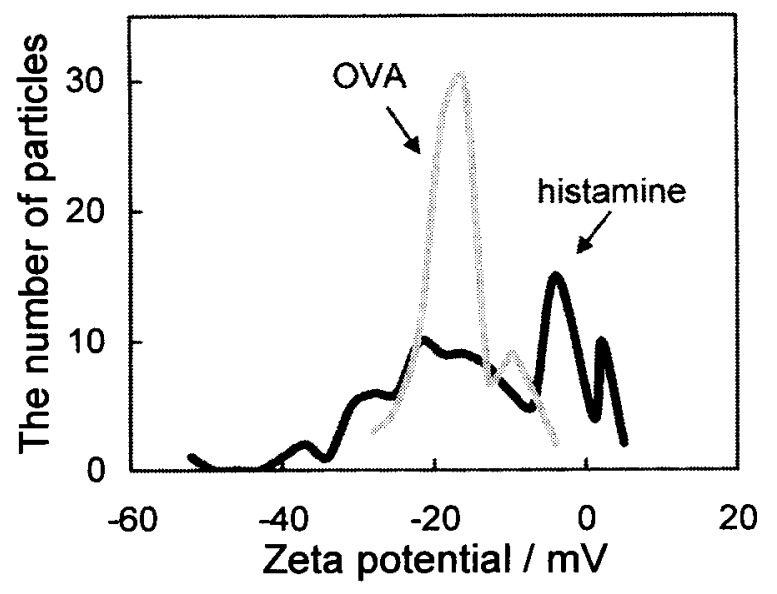

Fig. 9. Distributions of zeta potential of OVA and histamine.

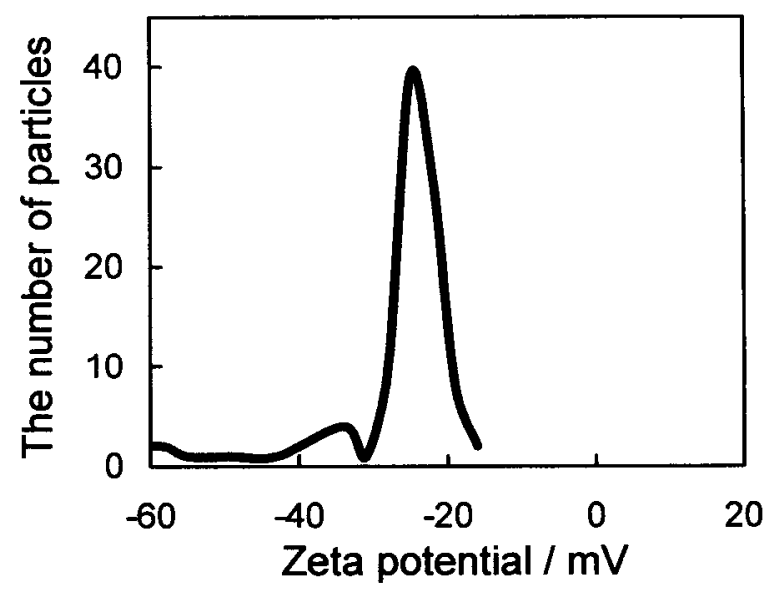

Fig. 10. Distribution of zeta potential of silica particles synthesized by ICP plasma. 
BSA derived by the theoretical calculations were about $\mathrm{pH} 10$ and $\mathrm{pH} 6$. So, cytochrome $\mathrm{C}$ may have a positive surface electric charge in $\mathrm{pH} 6$, and BSA may not have an electric charge. On the other hand, the isoelectric point of OVA determined by theoretical calculation was about $\mathrm{pH} 5$, so OVA may have a negative electric charge in $\mathrm{pH} 6$. In fact, the results of measurement of zeta potential show that OVA has a negative electric charge in $\mathrm{pH} 6$. Additionally, the isoelectric point of silica particles is about $\mathrm{pH} 2$; this value shows that silica particles may have a negative surface electric charge.

Therefore, we consider that the adsorption ability of silica particles for biotic substances is connected to electrostatic interactions. Thus, cytochrome $\mathrm{C}$ with a positive surface electric charge was supposed to be adsorbed to silica particles with negative surface electric charge. Actually the cytochrome $\mathrm{C}$ was adsorbed. Also the silica particle synthesized by ICP plasma had a negative surface electric charge.

We considered that the electrostatic interaction dominates the adsorption process between silica particles and some proteins. Next, we decided to look for some other biotic substances which may be adsorbed to silica particles by the concept of electrostatic interaction. Thus, a biotic substance which has a positive surface electric charge in $\mathrm{pH} 6$ was found. Histamine was applied to the condition. Histamine is a kind of neurotransmitter; it brings on itching. If the histamine at a corium could be drawn toward beneath the skin by the electrostatic interaction between histamine and silica particles on the skin, histamine can not attach itself to receptors which lie between corium and epidermides. Then if the itchiness was relieved, silica particles may be used as an anti-itch cream.

So we evaluated the adsorption ability of silica particles for histamine. As a result, histamine was adsorbed by silica particles, and the amount of adsorbed histamine had a proportional connection with the histamine concentration of solution. Then, the amount of adsorbed histamine was about $13 \%$ of the original amount of histamine in the buffer solution. On the other hand, the percentage of his- tamine particles which had a positive surface electric charge in $\mathrm{pH} 6$ was about $12 \%$. So it is considered that histamine particles with positive surface electric charge were adsorbed by silica particles with negative surface electric charge. From this result also we considered that the adsorption ability of silica particles is dominated by the electrostatic interactions.

\section{Conclusion}

We synthesized silica nano powder using ICP plasma. Formed silica particles showed adsorption ability for some biotic substances such as BSA, cytochrome $\mathrm{C}$, and histamine. On the other hand, silica particles show no adsorption for OVA. The results can be explained in terms of the surface electric charge data obtained by measurements of zeta potential. Silica particles have a negative surface electric charge in $\mathrm{pH}$ 6. So, the substances which have a positive surface electric charge were adsorbed by silica particles. On the other hand, the substance which has a negative surface electric charge was not adsorbed. We considered that electrostatic interactions dominate the adsorption process between silica particles and biotic substances.

From now, the same adsorption experiment will be performed using silica particles synthesized by different processes, and results will be compared to the results of silica particles synthesized using ICP plasma. Also, the behavior of silica particles in vivo is going to be evaluated. We aim for the clarification of the mechanism of adsorption ability by silica particles for biotic substances, and seek for applications of silica particles synthesized using ICP plasma as adsorbents for proteins or as anti-itch cream.

\section{References}

1. J. J. Kirkland, Journal of Chromatography 83 (1973) 149.

2. S. Oh, T. Ishigaki, Thin Solid Films 457 (2004) 186. 